HNO 2021 $69: 156$

https://doi.org/10.1007/s00106-020-00987-x Online publiziert: 13. Januar 2021

(c) Springer Medizin Verlag GmbH, ein Teil von Springer Nature 2021

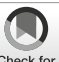

\title{
Florian Simon
}

Klinik und Poliklinik für Hals-Nasen-Ohrenheilkunde, LMU Klinikum, München, Deutschland

\section{Erratum zu: Diagnostik und Behandlung der Otitis externa}

Erratum zu:

HNO 2020

https://doi.org/10.1007/s00106-020-00

952-8

Hinweis: Teile dieses Beitrags wurden bereits in der Zeitschrift MMW Fortschritte der Medizin (2019) 161:35-39 erstpubliziert.

Ergänzung auf S. 883 im Abschnitt „Therapie der akuten diffusen Otitis externa“: Für die Salbenstreifeneinlage steht neben der Wirkstoffkombination Fluocinolonacetonid und Neomycin im „Off-Label-Use“ ebenso die Kombination aus Polymyxin-B-Sulfat, Bacitracin und Hydrocortisonacetat als zugelassene Alternative für die Indikation der Otitis externa diffusa zur Verfügung.

Wir bitten, den Hinweis und die Ergänzung zu beachten.

\section{Korrespondenzadresse}

Dr. med. Florian Simon

Klinik und Poliklinik für Hals-Nasen-

Ohrenheilkunde, LMU Klinikum

Marchioninistr. 15, 81377 München,

Deutschland

florian.simon@med.uni-muenchen.de
Die Online-Version des Originalartikels ist unter https://doi.org/10.1007/s00106-020-00952-8 zufinden. 